Abstract

\title{
Genistein in Prostate Cancer Prevention and Treatment ${ }^{\dagger}$
}

\section{Omer Kucuk}

Winship Cancer Institute, Emory University, Atlanta, GA 30322, USA; omer.kucuk@emory.edu

+ Presented at the 3rd International conference on Natural Products for Cancer Prevention and Therapy, Kayseri, Turkey, 18-20 December 2019.

Published: 9 February 2020

\begin{abstract}
Botanical compounds have been found to modulate genetic and epigenetic pathways of cancer development and progression. We have studied nutritional interventions, with emphasis on soy isoflavones. Preclinical and clinical translational research have been conducted investigating the potential use of natural compounds, particularly soy isoflavones, in the prevention and treatment of cancer. Clinical trials with soy isoflavones in prostate cancer patients have shown that they are potent anti-cancer agents that may be useful in preventing and slowing the progression of prostate cancer. Soy isoflavones could also prevent chemotherapy and radiation therapy toxicities. Furthermore, soy isoflavones may enhance the efficacy of chemotherapy and radiation therapy in patients with prostate cancer. Soy food intake has been associated with a low risk of several cancers. In addition, soy food consumption during cancer treatment may result in better outcomes and longer survival. These observations led to in vitro and in vivo mechanistic studies to elucidate the biological actions of various compounds in soybeans. Soy isoflavones have been found to have profound biological effects and modulate many of the pathways involved in cancer development and progression. In addition to their selective estrogen receptor modulatory effects, these compounds have anti-oxidant, anti-inflammatory and epigenetic effects, which may explain their potential role in cancer prevention and treatment. Soy foods and soy isoflavones can be easily taken together with conventional cancer treatments such as surgery, radiation, chemotherapy, hormone therapy, targeted agents and immunotherapeutic agents. They may enhance the efficacy and reduce the toxicities of radiation therapy, chemotherapy, hormone therapy and other conventional cancer treatments. Natural products such as soy isoflavones could be used to improve treatment effects and quality of life of patients. Soy isoflavones should be investigated in symptom control, quality of life, palliative care and survivorship research.
\end{abstract}

Keywords: soy isoflavones; genistein; prostate cancer 